\title{
Estudo comparativo sobre qualidade de vida, sobrecarga e sintomas musculoesqueléticos em cuidadores de idosos
}

\author{
Comparative study about quality of life, overload and musculoskeletal symptoms in \\ elderly caregivers
}

\author{
Estudio comparativo a cerca de la calidad de vida, sobrecarga y síntomas \\ musculoesqueléticos en cuidadores de ancianos
}

Laércio Bruno Ferreira Martins ${ }^{1 *}$, Caroline Rodrigues de Barros Moura ${ }^{1}$, Ana Flávia Machado de Carvalho ${ }^{1}$, Nayana Pinheiro Machado de Freitas Coelho ${ }^{1}$, Maura Cristina Porto Feitosa ${ }^{1}$, Jordano Leite Cavalcante de Macêdo ${ }^{1}$, Veruska Cronemberger Nogueira Rebêlo ${ }^{1}$.

\section{RESUMO}

Objetivo: Comparar Qualidade de Vida, sintomas osteomioarticulares e sobrecargas em cuidadores de idosos. Métodos: Trata-se de um estudo epidemiológico, observacional, descritivo, transversal e quantitativo, cuja amostra foi composta por setenta e seis indivíduos de ambos os sexos e com idade entre 18 e 55 anos, alocados em dois grupos, cuidadores formais (Grupo A) e cuidadores familiares (Grupo B). Os dados foram coletados com os questionários WHOQOL-BREF, QNSO e EZBI. Resultados: A Qualidade de Vida Geral (QVG) foi maior no Grupo A que no B. A sobrecarga leve concentrou-se majoritariamente no Grupo B e a sobrecarga grave no Grupo A. Nos sintomas musculoesqueléticos, o Grupo A sofreu acometimentos de região lombar, dorsal e ombros. Houve afastamentos por acometimento na região lombar, dorsal e ombros. No Grupo B, houve acometimentos de região lombar, ombros e dorsal. Não ocorreram afastamentos neste grupo, apesar de ter sofrido maiores sobrecargas e ter sido mais exposto a sintomas musculoesqueléticos e menor QVG, por executar suas atividades de forma anônima, sem vínculo formal no desempenho de suas funções. Conclusão: O Grupo A sofreu menos desgastes, afastando-se mais de atividades laborais, enquanto o Grupo B sofreu mais desgastes e sobrecargas, contudo com ausência de afastamentos.

Palavras-chave: Qualidade de vida, Sobrecarga, Saúde do trabalhador, Doenças musculares, Cuidadores.

\begin{abstract}
Objective: To compare Quality of Life, musculoskeletal symptoms and overloads in caregivers of the elderly. Methods: This is an epidemiological, observational, descriptive, cross-sectional and quantitative study, whose sample consisted of seventy-six individuals of both sexes, aged between 18 and 55 years, allocated in two groups, formal caregivers (Group A) and family caregivers (Group B). Data were collected with the WHOQOLBREF, QNSO and EZBI questionnaires. Results: General Quality of Life (GQL) was higher in Group A than in B. Light overload was mostly concentrated in Group B and severe overload in Group A. In musculoskeletal symptoms, Group A suffered lumbar, dorsal and shoulder involvement. There were absences due to lumbar, dorsal and shoulder involvement. In Group B, there were lower back, shoulder and back injuries. There were no absences in this group, despite having been overloaded and being more exposed to musculoskeletal symptoms and lower QOL, maybe they performed their activities anonymously, without formal ties in the performance of their functions. Conclusion: Group A suffered less wear and tear, moving away from work activities, while Group B suffered more wear and tear, however with no absence from work.
\end{abstract}

Keywords: Quality of life, Overload, Occupational health, Muscular diseases, Caregivers.

${ }^{1}$ Universidade Estadual do Piauí (UESPI), Teresina-Piauí. "E-mail: laerciom42@gmail.com 


\section{RESUMEN}

Objetivo: Comparar la calidad de vida, los síntomas musculoesqueléticos y la carga en los cuidadores de los ancianos. Métodos: Este es un estudio epidemiológico, observacional, descriptivo, transversal y cuantitativo, cuya muestra consistió en setenta y seis individuos de ambos sexos, con edades comprendidas entre 18 y 55 años, asignados en dos grupos, cuidadores formales (Grupo A) y cuidadores familiares (Grupo B). Los datos fueron recolectados con los cuestionarios WHOQOL-BREF, QNSO y EZBI. Resultados: Calidad de vida general (QVG) fue mayor en el Grupo A que en B. La sobrecarga ligera se concentró principalmente en el Grupo B y la sobrecarga severa en el Grupo A. En los síntomas musculoesqueléticos, el Grupo A sufrió afectación lumbar, dorsal y del hombro. Hubo ausencias por afectación lumbar, dorsal y del hombro. En el Grupo B, hubo lesiones en la espalda baja, hombros y espalda. No hubo ausencias en este grupo, a pesar de haber estado sobrecargado y estar más expuesto a los síntomas musculoesqueléticos y a reducir la calidad de vida, pues realizaron sus actividades de forma anónima, sin vínculos formales en el desempeño de sus funciones. Conclusión: el Grupo A sufrió menos desgaste, alejándose de las actividades laborales, mientras que el Grupo B sufrió más desgaste, sin embargo, sin ausencia del trabajo.

Palabras clave: Calidad de vida, Sobrecarga, Salud laboral, Enfermedades musculares, Cuidadores.

\section{INTRODUÇÃO}

Estima-se que existam atualmente no Brasil cerca de 20,5 milhões de pessoas acima de 60 anos, e há projeções que este número seja de pelo menos dois bilhões de pessoas no mundo até o ano 2050, com concentração principalmente em países em desenvolvimento. Estes indivíduos, em franco processo de envelhecimento, ficam expostos à ocorrência de doenças crônicas não transmissíveis e outros fatores que podem gerar dependências parciais ou totais. Neste âmbito, a figura do cuidador entra em cena, podendo estes serem familiares ou formais (CARRARO PFH, 2016; COPETTI LC, et al., 2019; MENDES PN, et al., 2019).

O processo de envelhecimento populacional no Brasil iniciou-se a partir de 1960, com rápido avanço, sendo estimado atualmente que nos próximos 20 anos esse número triplique, e esse aumento traz consigo vários fatores como declínios físico e mental, condições que afetam não somente o idoso, como aqueles que estão incluídos no seu processo de envelhecimento. Idosos com síndromes demenciais ou condições como a manifestação de doenças crônicas, apresentam deterioração clínica mais rápida que os demais, o que exige esforço de seus cuidadores (CARRARO PFH, 2016; LOPES RA, et al., 2013; DULLIUS WR e MIROTT AMBA, 2016).

Os cuidadores familiares geralmente são constituídos por filhos, netos ou familiares próximos. Prover assistência domiciliar e integral ao idoso torna-se por vezes uma realidade complexa, onde o cuidador é submetido a uma grande carga negativa quanto ao psicológico, físico, e por vezes, pelo isolamento social. Estes fatores geralmente se associam ao fato destes cuidadores não possuírem o preparo adequado para exercer tal função, muitas vezes não tem acesso sobre maneiras corretas de manejar o idoso, não desfrutam de carga horária definida, há um grande envolvimento emocional com o familiar, entre outros fatores que podem desencadear o surgimento de situações como depressão, ansiedade, medo, insegurança, cansaço e dores físicas que podem evoluir para casos mais graves (CARRARO PFH, 2016; CARRILO MGC, et al., 2013; CARVALHO MS e MARTINS JCA, 2016; GARCÍA EPL, 2016; MENDES PN, et al., 2019).

Já os cuidadores formais são profissionais que prestam auxílio a idosos à domicilio ou em Instituições de Longa Permanência para Idosos (ILPI). Geralmente têm acesso a cursos, treinamentos e outras estratégias para desempenhar a função, possuem carga horária definida e gozam dos direitos propostos por qualquer pessoa com vínculo empregatício. Apesar do fator emocional estar menos evidente, o cuidador formal pode desenvolver vínculos afetivos com o idoso, atentando ainda para o fato que mesmo com preparo, o manejo com o idoso pode acarretar dores físicas e levar a desgastes e problemas osteomioarticulares (ALENCAR MCB, et al., 2010; CARRILO MGC, et al., 2013; CAVALCANTI AD, 2013; CARVALHO MS e MARTINS JCA, 2016). 
Desta forma, a assistência ao idoso é um trabalho árduo e que exige esforços múltiplos dos cuidadores, sejam estes familiares ou formais, podendo de certa forma, repercutir em vários âmbitos da vida dos mesmos. Assim, este estudo objetivou estudar e comparar as repercussões sobre qualidade de vida (QV), sintomas osteomioarticulares e possíveis sobrecargas sofridas por este público.

\section{MÉTODOS}

Trata-se de um estudo epidemiológico, observacional, descritivo, transversal e quantitativo, em que foram incluídos indivíduos de ambos os sexos, sendo estes, cuidadores familiares e formais de uma cidade do estado do Piauí.

Foram incluídos na pesquisa pessoas que desempenhavam a atividade de cuidador de idosos há pelo menos 6 meses, com idade entre 18 e 55 anos. Foram excluídos questionários preenchidos incorretamente e participantes que não assinaram o Termos de Consentimento Livre e Esclarecido (TCLE).

Este estudo obedeceu aos critérios éticos com base na Resolução 466/12 do Conselho Nacional de Saúde (CNS). A sua realização deu-se após aprovação pelo Comitê de Ética em Pesquisa (CEP) da Universidade Estadual do Piauí - UESPI, com o n² 2.624.033 e autorização das instituições coparticipantes.

A coleta dos dados foi realizada no período de setembro de 2018 a maio de 2019. Os cuidadores formais (Grupo A) foram abordados nas ILPI nas quais trabalhavam. Os cuidadores familiares (Grupo B) foram contatados em suas respectivas residências, sendo explicados e esclarecidos os procedimentos da pesquisa e leitura do TCLE, iniciando-se posteriormente a entrevista, realizada em local reservado em ambos os casos. Todos os participantes assinaram o TCLE ao concordarem em participar do estudo.

Os questionários aplicados na pesquisa, foram identificados por números, não havendo, dessa forma, nenhum meio de identificação nominal dos mesmos. Foram utilizadas três ferramentas nas entrevistas:

\section{Questionário de qualidade de vida da organização mundial da saúde abreviado (WHOQOL-BREF)}

Questionário constituído por 26 perguntas, sendo as perguntas número 1 e 2 sobre a Qualidade de Vida Geral (QVG). As respostas seguem uma escala de Likert, de 1 a 5, onde quanto maior a pontuação, melhor a QV. Excetuando-se as duas questões iniciais, o instrumento possui 24 facetas, que compõem 4 domínios: Físico, Psicológico, Relações Sociais e Meio Ambiente (WACHHOLZ PA, et al., 2013).

\section{Escala zarit burden interview (EZBI)}

Escala que permite avaliar a sobrecarga objetiva e subjetiva do cuidado informal e que inclui informações sobre saúde, vida social, vida pessoal, situação financeira, situação emocional e tipo de relacionamento. Cada item é pontuado de forma qualitativa/quantitativa da seguinte forma: nunca $=(1)$; quase nunca $=(2)$; às vezes $=(3)$; muitas vezes $=(4)$ e quase sempre $=(5)($ SEQUEIRA CACS, 2010$)$.

\section{Questionário nórdico de sintomas osteomusculares (QNSO)}

Questionário que avalia os sintomas osteomusculares em pescoço, ombro, cotovelo, antebraço, punho/mão/dedos, região dorsal, região lombar, quadril/coxa, joelho, tornozelo/pé, e consiste em escolhas múltiplas ou binárias quanto à ocorrência de sintomas nas diversas regiões anatômicas nas quais são mais comuns. O respondente deve relatar a ocorrência dos sintomas considerando os 12 meses e os últimos sete dias precedentes à entrevista, bem como relatar a ocorrência de afastamento das atividades rotineiras no último ano (PINHEIRO FA, et al., 2002).

Para o tratamento estatístico os dados foram organizados em planilhas do programa Microsoft@ Office Excel $₫ 2010$ e distribuídos conforme os objetivos estabelecidos. Posteriormente, a estes dados foram aplicados os testes de Kolmogorov-Smirnov para verificação de aderência à normalidade, o teste T de Student para amostras independentes e comparação de médias e o teste de Correlação de Pearson para sintomas e afastamentos. As análises foram realizadas no Software IBM® Stastitical Package fot the Social Sciences (SPSS) versão 19, sendo adotado nível de confiança de 95\% para todos os testes estatísticos e fixado valor de $p<0,05$ para rejeição de hipótese nula. 


\section{RESULTADOS E DISCUSSÃO}

Neste estudo foram entrevistados 98 participantes, sendo 58 cuidadores formais e 40 cuidadores familiares, contudo, foram elegíveis 73 indivíduos, divididos em dois grupos: Grupo A = 46 e Grupo B = 27 .

$\mathrm{Na}$ caracterização amostral do Grupo A o sexo feminino representava $50 \%(n=23)$ dos indivíduos. No que se refere à faixa etária, 43,5\% $(n=20)$ possuíam de 22 a 35, 34,8\% $(n=16)$ de 36 a 45 e $21,7 \%(n=10)$ de 46 a 55 anos. Relacionado ao nível de escolaridade, $76,9 \%(n=30)$ possuíam segundo grau, $10,3 \%(n=4)$ nível técnico, $7,7 \%(n=3)$ primeiro grau e $5,1 \%(n=2)$ terceiro grau. Com relação ao estado civil, $60 \%(n=24)$ dos cuidadores eram casados ou viviam em união estável, 35\% $(n=18)$ eram solteiros e $5 \%(n=2)$ divorciados. Neste grupo, 39,1\% ( $n=18)$ dos indivíduos não possuíam filhos, 36,9\% ( $n=17)$ possuíam até 2 filhos e 23,9\% ( $n=11$ ) possuíam de 3 a 4 filhos. Em relação ao tempo como cuidador, $40 \%$ ( $n=18)$ cuidavam há pelo menos 1 a 3 anos, 26,7\% (n=12) de 7 a 10 anos, 20\% (n=9) há mais de 10 anos e 13,3\% (n=6) de 4 a 6 anos, sendo esta, a atividade exclusiva de $71,8 \%(n=28)$ dos indivíduos. No tocante ao turno de trabalho, $82 \%, 6 \%(n=38)$ dos indivíduos trabalhavam em período matutino e vespertino, enquanto $17,4 \%(n=8)$ em período noturno.

O Grupo B foi caracterizado por $70,4 \%(n=19)$ de indivíduos do sexo feminino e $29,6 \%(n=8)$ do sexo masculino. No tocante ao grau de parentesco, $70,4 \%(n=19)$ eram filhos dos idosos a quem prestavam cuidado, $14,8 \%(n=4)$ netos e 3,7\% $(n=1)$ sobrinhos. Concernente à faixa etária, $59,3 \%(n=16)$ possuíam entre 46 e 55 anos, $29,6 \%(n=8)$ entre 22 e 35 anos e $11,1 \%(n=3)$ entre 36 e 45 anos de idade. Relacionado ao nível de escolaridade, $55,6 \%(n=15)$ possuíam segundo grau, $22,2 \%(n=6)$ terceiro grau, $14,8 \%(n=4)$ primeiro grau e 3,7\% (n=1) possuíam respectivamente nível técnico ou não possuíam nenhum grau de instrução.

Referente ao estado civil, 40,7\% ( $n=11)$ eram casados ou estavam em uma união estável, $37 \%(n=10)$ eram solteiros e $22,2 \%(n=6)$ divorciados. Com relação ao número de filhos, $48,1 \%(n=13)$ não possuíam filhos, $37 \%(n=10)$ possuíam até 2 filhos e $11,1 \%(n=3)$ possuíam de 3 a até 4 filhos. Neste grupo, 38,5\% $(\mathrm{n}=10)$ dos indivíduos acompanhavam o idoso por um período de 6 a 10 anos, 26,1\% $(n=6)$ de 1 a 5 anos ou 11 a 15 anos, respectivamente. Outros $15,4 \%(n=4)$ acompanhavam o idoso por um período superior a 15 anos. Destes cuidadores, $55,6 \%(n=15)$ dedicavam-se exclusivamente à atividade de cuidar. Neste grupo foi observada também a divisão de cuidados, sendo que $62,9 \%(n=17)$ dos entrevistados realizavam a divisão de cuidados e $37 \%(n=10)$ afirmaram não realizar.

Ambos os grupos prestavam assistência a idosos com idades entre 60 e 108 anos com dependência em variados níveis em decorrência de Alzheimer e outras demências, deficiência de sentidos, amputações, sequelas neurológicas e distúrbios musculoesqueléticos. A Agência Nacional de Vigilância Sanitária (ANVISA) define três graus de dependência em idosos: Grau I - Idosos independentes, mesmo que necessitem equipamentos de auto-ajuda; Grau II - Idosos que necessitem ajuda em até três atividades de cuidados próprios, como alimentação mobilidade e higiene; e Grau III: Idosos que requeiram assisitência em todas as atividades de autocuidado para a vida diária ou com comprometimento cognitivo (BRASIL, 2005).

Segundo Anjos KF (2018) e Martins G, et al. (2019) o predomínio do sexo feminino neste estudo e em estudos semelhantes é atribuído ao fato de que as mulheres são propensas a uma maior expectativa de vida que homens e também porque o desempenho da atividade exige ampla dedicação física e emocional. Por outro lado, outros autores atribuem em seus respectivos estudos a maior presença feminina no papel de cuidadora em decorrência do contexto histórico, onde a mulher sempre teve sua função social vinculada ao ato de cuidar, enquanto o homem desempenhava o papel de provedor de mantimentos para a sobrevivência (SAMPAIO LS, et al., 2018; e DOS SANTOS MESSIAS LA, 2018).

A concentração predominante de indivíduos jovens e o predomínio de segundo grau de escolaridade foram semelhantes aos encontrados em outros estudos semelhantes, contudo, nestes estudos foi realizada correlação entre a QV e a idade dos cuidadores, demonstrando que cuidadores jovens apresentam menor QV em relação a cuidadores com mais idade, o que não foi encontrado no estudo atual (QUEIROZ RS, et al., 2018).

Com relação ao tempo de cuidado e ao desempenho de outra atividade, os resultados encontrados no atual estudo foram semelhantes aos de outros estudos com a mesma temática, realizados com cuidadores 
de idosos com demências. Estes estudos demonstraram que a média do tempo de cuidado prestado foi de $47,9 \pm 34,8$ meses, sendo que metade de sua amostra desempenhava esta atividade em tempo integral e $60 \%$ realizava a divisão do cuidado.

Contudo, o mesmo autor infere que o tempo dispendido no cuidado tende a se intensificar quando o cuidador é residente no mesmo domicílio com o paciente (DOS SANTOS MESSIAS LA, 2018). Na Tabela 1 são descritos os resultados da avaliação da qualidade de vida de ambos os grupos se utilizando o instrumento WHOQOL-BREF.

Tabela 1 - Comparação dos domínios do questionário WHOQOL-BREF, entre grupos de cuidadores familiares e formais em Teresina-PI. Teresina, 2019.

\begin{tabular}{cccccc}
\hline \multicolumn{1}{c}{ Domínios } & Grupo & N & M & DP & p-valor $^{*}$ \\
\hline \multirow{2}{*}{ Qualidade de vida geral } & A & 46 & 3,98 & 0,70 & \multirow{2}{*}{0,004} \\
Domínio físico & B & 27 & 3,46 & 0,73 & \\
& A & 46 & 4,10 & 0,48 & $<0,0001$ \\
Domínio psicológico & B & 27 & 3,65 & 0,53 & \\
Domínio relações sociais & A & 46 & 4,10 & 0,43 & $<0,0001$ \\
Domínio meio ambiente & B & 27 & 3,63 & 0,58 & 0,004 \\
& A & 46 & 4,07 & 0,72 & \\
& B & 27 & 3,53 & 0,77 & 0,013 \\
\hline
\end{tabular}

Legenda: Grupo A: Cuidadores Formais; Grupo B: Cuidadores Familiares; N: Amostra; M: Média; DP: Desvio Padrão; " $p$-valor obtido pelo teste t de Student. Fonte: Martins LBF, et al., 2019.

Seguindo o mesmo raciocínio já proposto por Anjos KF (2018) e Queiroz RS, et al. (2018), o aumento progressivo da sobrecarga aumenta na mesma proporção que o comprometimento da QV. Esse comprometimento pode estar ou não correlacionado às atividades assistenciais oferecidas ao idoso, que somadas a outras atividades cotidianas do cuidador, exigem uma readequação e reestruturação do ambiente domiciliar, uma vez que o mesmo, muitas vezes precisa abdicar de suas atividades laborais e sociais em prol de uma maior assistência ao seu ente.

No estudo de Queiroz RS, et al. (2018), realizado com 81 cuidadores formais e familiares os domínios Físico e Relações Sociais foram os melhores avaliados, enquanto que domínios Psicológico e Meio Ambiente tiveram menores pontuações.

Já no estudo de Anjos KF (2018), que se utilizou de uma amostra de 71 cuidadores familiares o escore do Índice Geral da Qualidade de Vida foi de 55,6 pontos, sendo o maior escore pontuado pelo domínio Físico $(56,4)$ e o menor pelo domínio Meio Ambiente $(40,8)$. No atual estudo, contudo foram encontrados dados parcialmente divergentes, onde os domínios Físico e Psicológico foram os melhor avaliados.

Dentre os outros fatores que podem influenciar neste grau de comprometimento da QV, pode-se citar a carga horária dispendida no cuidado e o grau de dependência do doente, sobretudo nos cuidadores que residem com o idoso, a ausência de apoio e informações adequadas e o grau de cansaço.

Além disso, há ainda outro fator de extrema relevância levantado por Sampaio LS, et al. (2018) e Anjos KF (2018), o suporte ofertado por terceiros na forma da divisão de cuidados, que quando presente contribui para uma melhor QV, e quando ausente contribui para o declínio da saúde física, social e mental dos cuidadores, afetando também a qualidade dos cuidados prestados por estes. 
Essa ausência de suporte ocorre porque quando um cuidador assume a responsabilidade do cuidado para si, outros possíveis cuidadores apresentam uma tendência de afastamento.

Prado AS, et al. (2017), citam ainda a existência de relações afetivas entre cuidadores familiares e idosos, que podem influenciar nos níveis de cansaço emocional e despersonalização. Em cuidadores formais, contudo, este aspecto torna-se menos evidenciado, ficando restrito ao caráter moral e profissional.

Entretanto, ainda assim, cuidadores formais podem sofrer com declínio da QV devido ao esgotamento profissional, que se evidencia conforme os anos trabalhados e também as horas de serviço, podendo evoluir de forma aguda ou estabelecer-se de forma crônica, mesmo naqueles cuidadores que deixam de desempenhar a atividade.

Na Tabela 2 é realizada a comparação intergrupo dos níveis de sobrecarga encontrados em ambos os grupos com o instrumento Escal Zarit Burden Interview.

Tabela 2 - Comparação dos domínios do instrumento Escala Zarit Buden Interview entre cuidadores familiares e formais de idosos em Teresina-PI. Teresina, 2019.

\begin{tabular}{cccccccc}
\hline & \multicolumn{3}{c}{ Grupo A } & \multicolumn{3}{c}{ Grupo B } & p-valor* \\
\hline Leve & $\mathrm{N}$ & $\mathrm{M}$ & $\mathrm{DP}$ & $\mathrm{N}$ & $\mathrm{M}$ & $\mathrm{DP}$ & \\
Moderada & 41 & 9,98 & 2,35 & 13 & 9,92 & 2,40 & 0,001 \\
& 4 & 17,00 & 2,00 & 10 & 16,70 & 1,89 & 0,001 \\
Grave & 1 & 26,00 & - & 4 & 27,75 & 2,63 & 0,001
\end{tabular}

Legenda: Grupo A: Cuidadores Formais; Grupo B: Cuidadores Familiares; N: Amostra; M: Média; DP: Desvio Padrão; * $p$-valor obtido pelo teste t de Student. Fonte: Martins LBF, et al., 2019.

Os níveis de sobrecarga encontrados neste estudo foram próximos aos encontrados no estudo de Diniz et al., 2018, que relatam sobrecarga tanto em cuidadores familiares quanto institucionalizados, contudo, em cuidadores familiares houve maiores índices de sobrecarga moderada $(25,7 \%)$ e sobrecarga alta $(14,3 \%)$, sendo o desconforto emocional da mesma forma menos presente em cuidadores formais que em familiares. Estes autores atribuem tais resultados à gravidade da doença, ao despreparo para o seu enfrentamento da situação, ao baixo nível de independência e convivência contínua com o idoso.

Por outro lado, Flesch LD, et al. (2017) realizaram um estudo buscando explicar os aspectos que deixam os cuidadores de idosos em situação de vulnerabilidade, demonstrando que além das obrigações já atribuídas ao cuidador, que por si só já são potencialmente estressantes, há outras situações agravantes como a sobreposição de papéis pela necessidade do desempenho de atividades concomitantes relacionadas a atividades laborais, cuidado de crianças pequenas e condições de saúde própria, que exacerbam a percepção de sobrecarga.

Esta sobreposição de papéis normalmente é acompanhada de disfunções emocionais e biomecânicas, que apresentam como principais sintomas sensações álgicas, que podem ser resultantes tanto da condição intrínseca de saúde do cuidador, como da falta de condições no ambiente de trabalho.

Além da experimentação dolorosa, pode haver também o acometimento de músculos, tendões e articulações, que culminam por afetar a vida social desses indivíduos, quando muitas vezes, estes têm que se afastar de suas ocupações (VAZ LCS, et al., 2018).

$\mathrm{Na}$ Tabela 3 estão expressos os sintomas referidos por indivíduos de ambos os grupos de cuidadores nos últimos 12 meses e últimos sete dias, bem como a frequência relativa de afastamentos, de acordo com o QNSO. 
Tabela 3 - Frequência de sintomas e afastamentos por região anatômica de cuidadores familiares e cuidadores formais de idosos segundo Questionário Nórdico de Sintomas Osteomioarticulares em TeresinaPI. Teresina, 2019.

\begin{tabular}{|c|c|c|c|c|c|c|c|}
\hline \multirow{2}{*}{ Região } & \multirow{2}{*}{ Grupos } & \multicolumn{2}{|c|}{$\begin{array}{c}12 \text { meses } \\
\text { precedentes }\end{array}$} & \multicolumn{2}{|c|}{$\begin{array}{c}7 \text { dias } \\
\text { precedentes }\end{array}$} & \multicolumn{2}{|c|}{$\begin{array}{l}\text { Afastamentos no último } \\
\text { ano }\end{array}$} \\
\hline & & $\mathbf{n}$ & $\%$ & $\mathbf{n}$ & $\%$ & $\mathbf{n}$ & $\%$ \\
\hline \multirow{2}{*}{ Pescoço } & $A$ & 7 & $15,2 \%$ & 6 & $13,0 \%$ & 1 & $2,2 \%$ \\
\hline & $B$ & 1 & $3,7 \%$ & 1 & $3,7 \%$ & 1 & $3,7 \%$ \\
\hline \multirow{2}{*}{ Ombros } & A & 13 & $28,3 \%$ & 6 & $13,0 \%$ & 4 & $8,7 \%$ \\
\hline & $B$ & 8 & $29,6 \%$ & 7 & $25,9 \%$ & 2 & $7,4 \%$ \\
\hline \multirow{2}{*}{ Cotovelo } & $A$ & 4 & $8,7 \%$ & 2 & $4,3 \%$ & 1 & $2,2 \%$ \\
\hline & $B$ & 3 & $11,1 \%$ & 2 & $7,4 \%$ & 0 & $0,0 \%$ \\
\hline \multirow{2}{*}{ Antebraço } & $A$ & 4 & $8,7 \%$ & 2 & $4,3 \%$ & 0 & $0,0 \%$ \\
\hline & $B$ & 3 & $11,1 \%$ & 1 & $3,7 \%$ & 0 & $0,0 \%$ \\
\hline \multirow{2}{*}{ Punhos/mãos/dedos } & A & 8 & $17,4 \%$ & 3 & $6,5 \%$ & 2 & $4,3 \%$ \\
\hline & $B$ & 4 & $14,8 \%$ & 5 & $18,5 \%$ & 0 & $0,0 \%$ \\
\hline \multirow{2}{*}{ Região dorsal } & $A$ & 10 & $21,7 \%$ & 7 & $15,2 \%$ & 3 & $6,5 \%$ \\
\hline & $B$ & 13 & $48,1 \%$ & 10 & $37,0 \%$ & 2 & $7,4 \%$ \\
\hline \multirow{2}{*}{ Região lombar } & $A$ & 16 & $34,8 \%$ & 10 & $21,7 \%$ & 9 & $19,6 \%$ \\
\hline & $B$ & 14 & $51,9 \%$ & 12 & $44,4 \%$ & 3 & $11,1 \%$ \\
\hline \multirow{2}{*}{ Quadris e coxas } & $A$ & 4 & $8,7 \%$ & 4 & $8,7 \%$ & 1 & $2,2 \%$ \\
\hline & $B$ & 1 & $3,7 \%$ & 0 & $0,0 \%$ & 0 & $0,0 \%$ \\
\hline \multirow{2}{*}{ Joelhos } & $A$ & 8 & $17,4 \%$ & 4 & $8,7 \%$ & 4 & $8,7 \%$ \\
\hline & $B$ & 4 & $14,8 \%$ & 4 & $14,8 \%$ & 0 & $0,0 \%$ \\
\hline \multirow{2}{*}{ Tornozelos } & $A$ & 5 & $10,9 \%$ & 4 & $8,7 \%$ & 3 & $6,5 \%$ \\
\hline & $B$ & 5 & $18,5 \%$ & 5 & $18,5 \%$ & 0 & $0,0 \%$ \\
\hline
\end{tabular}

Legenda: Grupo A: Cuidadores Formais; Grupo B: Cuidadores Familiares. Fonte: Martins LBF, et al., 2019.

No estudo de Vaz LCS, et al., (2018) foram relatadas maior frequência de dor entre a coluna lombar e região dorsal, tanto em cuidadores formais quanto familiares. No estudo de Diniz MAA, et al. (2018), os cuidadores formais também apontaram a lombalgia como o principal sintoma musculoesquelético.

Esses dados assemelham-se aos do estudo atual, contudo, apesar do acometimento dessas regiões anatômicas, não foi relatada nestes estudos ocorrência de incapacitação para o exercício das atividades de vida diária como o próprio ato de cuidar, diferentemente do que aconteceu no estudo atual.

Porém, no estudo atual, cuidadores formais apresentaram maior parcela de afastamentos devido a esses sintomas musculoesqueléticos, principalmente porque este grupo, dessemelhante do grupo familiar, possui mais suporte profissional, não podendo o cuidador familiar afastar-se do desempenho de sua atividade, uma vez que na maior parte do tempo a exerce de forma solitária.

Dessa forma, o autocuidado neste público fica marginalizado e muitas vezes, inexistente, de forma que a dor se torna um fator constantemente presente na vida desses indivíduos, podendo existir mesmo na ausência de causas identificadas (CESÁRIO VAC, et al., 2017; NIEROTKA RP e PORTELLA MR, 2017; VAZ LCS, et al., 2018).

Na Tabela 4 são realizadas as Correlações de Pearson entre os sintomas referidos nos últimos 12 meses e os sintomas referidos nos últimos 7 dias nas diversas regiões anatômicas em ambos os grupos. 
Tabela 4 - Correlações de Pearson entre sintomas e afastamentos para as diversas regiões anatômicas em cuidadores familiares e formais em Teresina-PI. Teresina, 2019.

\begin{tabular}{|c|c|c|c|c|c|c|}
\hline & \multicolumn{2}{|c|}{$\begin{array}{c}\text { Sintomas em } 12 \text { meses } x \\
\text { Sintomas } 7 \text { dias }\end{array}$} & \multicolumn{2}{|c|}{$\begin{array}{c}\text { Sintomas em } 12 \text { meses } x \\
\text { afastamentos }\end{array}$} & \multicolumn{2}{|c|}{$\begin{array}{l}\text { Sintomas } 7 \text { dias } x \\
\text { afastamentos }\end{array}$} \\
\hline & A & B & A & B & A & B \\
\hline Pescoço & $0,914^{\star *}$ & 0 & $0,352^{*}$ & 0 & $0,385^{\star \star}$ & 0 \\
\hline Ombros & $0,617^{\star *}$ & $0,727^{\star *}$ & $0,320^{*}$ & 0,126 & $0,339^{*}$ & 0,155 \\
\hline Cotovelo & $0,313^{*}$ & $0,800^{\star *}$ & $0,483^{* *}$ & 0 & $-0,032$ & 0 \\
\hline Antebraço & $0,691^{* *}$ & $0,555^{\star \star}$ & 0 & 0 & 0 & 0 \\
\hline Punhos/mãos/dedos & $0,576^{* *}$ & $0,875^{\star *}$ & $0,465^{\star *}$ & 0 & $0,807^{\star \star}$ & 0 \\
\hline Região dorsal & $0,804^{* *}$ & $0,796^{* *}$ & $0,501^{* *}$ & 0,294 & $0,623^{\star *}$ & 0,369 \\
\hline Região lombar & $0,722^{\star *}$ & $0,713^{\star *}$ & $0,675^{\star *}$ & 0,105 & $0,803^{\star *}$ & 0,158 \\
\hline Quadris e coxas & $0,726^{\star *}$ & 0 & $0,483^{* *}$ & 0 & $0,483^{\star \star}$ & 0 \\
\hline Joelhos & $0,673^{* *}$ & $0,707^{\star *}$ & $0,673^{* *}$ & 0 & $0,452^{* \star}$ & 0 \\
\hline Tornozelos & $0,884^{* *}$ & $0,755^{\star *}$ & $0,756^{* *}$ & 0 & $0,856^{\star *}$ & 0 \\
\hline
\end{tabular}

Legenda: Grupo A: Cuidadores Formais; Grupo B: Cuidadores Familiares; ${ }^{*}$ Correlação significativa pelo teste Correlação de Pearson. Fonte: Martins LBF, et al., 2019.

De acordo com Nierotka RP e Portella MR (2017), com o desempenho da função a longo prazo, o cuidador sofre interferência em sua percepção acerca de sua própria saúde, fato ligado à condição de dependência e precariedade de um suporte social efetivo, que repercute negativamente nos problemas de saúde adquiridos por estes indivíduos no exercício da função.

Constituindo-se em um ciclo vicioso, segundo Lopes RA, et al. (2013), porquanto, o cuidador com condições físicas e emocionais desfavoráveis apresenta sintomas musculoesqueléticos, depressivos e de ansiedade que interferem em sua própria QV e culminam em uma assistência inadequada e ineficiente ao idoso, posto que quando este cuidador apresenta tais sintomas, tende a avaliar a dor de seus pacientes de maneira distorcida, e, por conseguinte, prestar-Ihes cuidados inadequados.

O mesmo autor infere que tais acontecimentos podem levar o cuidador ao impedimento da realização de suas atividades normais. Por outro lado, outros estudos afirmam que essa baixa percepção acerca da própria saúde requer uma maior atenção por meio da criação de programas dedicados e de uma ampla rede de apoio, fundamentais para a redução da sobrecarga produzida pelo ato de cuidar.

Contudo, neste estudo, a alta correlação encontrada para afastamentos ocorridos no último ano em cuidadores formais pode refletir uma ineficiência no acesso a serviços de informação e suporte social, dado que a ocorrência do impedimento da realização de suas atividades ocorre quase sempre em estágios avançados de sobrecarga e desgaste.

Em contrapartida, a baixa correlação entre afastamentos e sintomas osteomioarticulares em cuidadores familiares no último ano, se justifica, pela ausência de assistência de terceiros e pelo desempenho da atividade de forma isolada. (NIEROTKA RP e PORTELLA MR, 2017; VAZ LCS, et al., 2018; FLESCH LD, et al., 2017)

Dentre as limitações deste estudo pode-se citar a não realização de cálculo estatístico para definição de amostra ideal, a subjetividade dos instrumentos utilizados e a amostra total de indivíduos final do estudo, limitada pelo grande número de desistências na participação. 


\section{CONSIDERAÇÕES FINAIS}

Este estudo demonstrou que cuidadores formais sofrem menos desgastes no exercício da função que os cuidadores familiares. Contudo, cuidadores formais ainda estão expostos a sintomas osteomioarticulares, sobrecarga e declínio da QV, sendo estes os indivíduos com mais afastamentos das atividades laborais. Por outro lado, os cuidadores familiares estiveram mais expostos a sobrecargas, sintomas osteomioarticulares e menor QV, não se afastando de suas atividades laborais, pela ausência de suporte de terceiros, estando impedido de dedicar-se a atividades de autocuidado. Dessa forma, pressupõe-se a necessidade de estudos adicionais com uma abordagem focada neste público, bem como a realização de políticas efetivas de educação em saúde e suporte a estes profissionais.

\section{REFERÊNCIAS}

1. ALENCAR MCB; et al. Musculoskeletal disorders and the care work of elderly in institutions, Fisioter. Mov.,2010; 23(1):63-72.

2. ANJOS KF; et al. Factors associated with the quality of life of family caregivers of elderly people. Ciencia y Enfermaria, 2018; 24(17): 185-199.

3. BRASIL, 2005. In: Biblioteca Virtual em Saúde do Ministérioo da Saúde - BVSMS. Brasília: Ministério da Saúde. Disponível em: http://bvsms.saude.gov.br/bvs/saudelegis/anvisa/2005/res0283_26_09_2005.html. Acesso em: 6 fev. 2020.

4. CARRARO PFH, et al. Qualidade de vida de cuidadores de idosos com diagnóstico de Alzheimer e o emprego de acupuntura - Revisão de Literatura. Mudança - Psicologia da Saúde, 2016; 24(02):65-70.

5. CARRILLO MGC; et al. Overload, anxiety and depression in caregivers of patients included in the home care program. Gerokomos, 2013; 24(03):120-123.

6. CARVALHO MS, MARTINS JCA. Palliative Care for Institutionalized Elderly Persons: Experience of Caregivers. Rev. Bras. Gerontol., 2016; 19(05):745-758.

7. CAVALCANTI AD; Envelhecimento e Institucionalização: uma revisão bibliográfica à luz da promoção da saúde. Rev. Kayrós Gerontologia, 2013; 16(4):159-174

8. CESÁRIO VAC; et al. Stress and quality of life of the family caregivers of elderly with Alzheimer's disease. Saúde Debate, 2017; 41(112):171-182.

9. COPPETTI LC; et al.; Scientific production of nursing on the family care of dependent elderly in the household. ABCS Health Sci., 2019; 44(1):58-66.

10. DINIZ MAA; et al. Comparative study between formal and informal caregivers of older adults. Ciência e Saúde coletiva, 2018; 23(1):3789-3798.

11. DOS SANTOS MESSIAS LA. Practical knowledge and life overload of caregivers of elderly with dementia. Sci Med., 2018; 28(3):1-8.

12. DULLIUS WR; MIROTT AMBA. Qualidade do Relacionamento do Familiar Cuidador com o Familiar Portador de Demência: revisão integrativa. Revista de Psicologia da IMED, 2016; 8(2):156-171.

13. FLESCH LD; et al. Psychological aspects of the quality of life of caregivers of the elderly: an integrative review. Geriatr Gerontol Aging., 2017; 11(3):38-49.

14. GARCÍA EPL. Cuidar a los que cuidan: los cuidadores informales. Revista Uruguaya de Enfermaría, 2016; 11(2):4958.

15. LOPES RA; et al. Caregivers of long-term care institutions for elderly: pain, anxiety and depression. Fisioterapia Brasil, 2013; 14(2):117-121.

16. MARTINS G. et al. Sociodemographic and health characteristics of formal and informal caregivers of elderly people with Alzheimer's Disease. Esc Anna Nery, 2019; 23(2):1-10.

17. MENDES PN; et al. Physical, emotional and social burden of elderly patients' informal caregivers. Acta Paul Enferm., 2019; 32(1):87-94.

18. NIEROTKA RP., PORTELLA MR. Perception of health and intensity of caregivers of pain associated with the elderly with Alzheimer time of caution. FisiSenectus, 2017; 05(2):03-12.

19. PINHEIRO FA; et al. Validity of the Nordic Musculoskeletal Questionnaire as morbidity measurement tool. Rev. Saúde Pública, 2002; 36(3):307-312.

20. PRADO AS; et al. Relationship between professional exhaust, quality of life and time of work in older caregivers. Revista Kairós - Gerontologia, 2017; 20(3):179-189.

21. QUEIROZ RS; et al. Sociodemographic profile and quality of life of caregivers of elderly people with dementia. Rev. Bras. Gerentol., 2018; 21(2):210-219.

22. SAMPAIO LS; et al. Quality of life and depression in caregivers of elderly dependents. Rev. APS, 2018; 21(1):112121.

23. SEQUEIRA CACS. Adaptação e Validação da Escala de Sobrecarga do Cuidador de Zarit. Revista Referência, 2010; 2(12):09-16.

24. VAZ LCS; et al. Health and work conditions between caregivers of elderly. Rev. Pesq. Fisio., 2018; 8(3):319-329.

25. WACHHOLZ PA; et al. Recognizing bueden and quality of life of family caregivers of frail elderly, Rev. Bras. Geriatr. Gerontol., 2013;16(3):513-526. 